\title{
Oral microbial community typing of caries and pigment in primary dentition
}

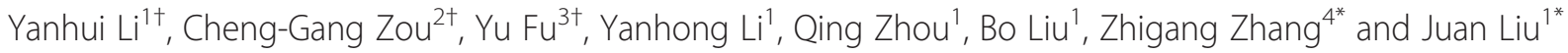

\begin{abstract}
Background: Black extrinsic discoloration in primary dentition is a common clinical and aesthetic problem that can co-occur with dental caries, the most common oral diseases in childhood. Although the role of bacteria in the formation of pigment and caries in primary dentition is important, their basic features still remain a further mystery.

Methods: Using targeted sequencing of the V1-V3 hypervariable regions of bacterial $16 \mathrm{~S}$ ribosomal RNA (rRNA) genes, we obtained a dataset consisting of 831,381 sequences from 111 saliva samples and 110 supragingival plaque samples from 40 patients with pigment (black extrinsic stain), 20 with caries (obvious decay), and 25 with both pigment and caries and from 26 healthy individuals. We applied a Dirichlet multinomial mixture (DMM)-based community typing approach to investigate oral microbial community types.

Results: Our results revealed significant structural segregation of microbial communities, as indicated by the identification of two plaque community types (A and B) and three saliva community types (C-E). We found that the independent occurrence of the two plaque community types, A and B, was potentially associated with our oral diseases of interest. For type A, three co-occurring bacterial genus pairs could separately play a potential role in the formation of pigment (Leptotrichia and Fusobacterium), caries (unclassified Gemellales and Granulicatella), and mixed caries and pigment (Streptococcus and Mogibacterium). For type B, three co-occurring bacterial genera (unclassified Clostridiaceae, Peptostreptococcus, and Clostridium) were related to mixed pigment and caries. Three dominant bacterial genera (Selenomonas, Gemella, and Streptobacillus) were linked to the presence of caries.
\end{abstract}

Conclusions: Our study demonstrates that plaque-associated oral microbial communities could majorly contribute to the formation of pigment and caries in primary dentition and suggests potential clinical applications of monitoring oral microbiota as an indicator for disease diagnosis and prognosis.

Keywords: Saliva, Supragingival plaque, Pigment, Caries, Oral microbiota

\section{Background}

Discovering the dynamics and relationships of human oral microbial community types will allow microbiologists to better associate changes in the microbiome with changes in health or disease. It is difficult to differentiate disease-associated microbiome changes from other factors by limiting inter-individual variation to the minimum amount, although the partitioning around meloids (PAM)based approach can capture various human gut microbial

\footnotetext{
* Correspondence: zhangzhigang@mail.kiz.ac.cn; liujuan1966@yahoo.com ${ }^{\dagger}$ Equal contributors

${ }^{4}$ State Key Laboratory of Genetic Resources and Evolution, Kunming Institute of Zoology, Chinese Academy of Sciences, Kunming 650223, China 'Department of Preventive \& Pediatric Dentistry, The Affiliated Stomatology Hospital of Kunming Medical University, Kunming, Yunnan 650100, China Full list of author information is available at the end of the article
}

enterotypes [1]. Recently, Ding \& Schloss employed an novel approach based on the Dirichlet multinomial mixture (DMM) model independent of the distance matrix [2]; this method significantly improved the microbial community typing across the human body that was performed by analyzing $16 \mathrm{~S}$ ribosomal RNA (16S rRNA) gene sequences [3]. For example, empirical comparison of the methods indicated that only two community types were identified in samples of saliva and supragingival plaque using the PAM-based approach, whereas four and six community types with the lowest Laplace values (for selecting the best number of metacommunities) were separately identified at the two oral sites using the DMM-based approach. These findings suggest that the DMM-based approach is superior for assessing the oral 
microbial community types associated with oral diseases, such as caries and pigment.

Caries is one of the most common oral diseases in childhood, and it results from many factors, including bacterial infection [4]. Recent studies have demonstrated that altered oral microbiota may be associated with caries [5-7]. However, oral microbial communities vary in a spatiotemporal manner among populations $[8,9]$. Thus, the microbial configurations associated with caries, a polymicrobial disease [10], during the same dentition stage remain poorly understood.

Black extrinsic discoloration in primary dentition is another common clinical and aesthetic problem in childhood that can co-occur with dental caries [11-15]. Black stain is considered a form of dental plaque that differs from other types by insoluble iron salts and high calcium and phosphate contents [16-18]. In particular, an association between black tooth stain and bacteria, such as actinomyces, has been reported [19-22]. However, how microbial assemblies in both saliva and plaque are linked to pigment (represented by black extrinsic stain) and to the presence of a combination of pigment and caries remains largely unknown.

To understand the role of bacteria in pigment (black extrinsic stain) formation and its co-occurrence with caries, we performed a large-scale molecular analysis of $16 \mathrm{~S}$ rDNA sequences using a DMM-based community typing approach to address two crucial issues: whether there are significant divergences of microbial communities associated with caries and/pigment within specific oral niches and what are the dominant constituents that potentially contribute to the occurrence of caries, pigment and their combination?

\section{Methods}

\section{Patient selection and sample collection}

Through visual inspection by a single investigator (Y.HG.L.), a specialist in pediatric dentistry, the status of caries was determined according to the International Caries Detection and Assessment System (ICDAS-II) [23], and black extrinsic tooth stain was evaluated based on the presence of pigmented dark lines parallel to the gingival margin or an incomplete coalescence of dark dots rarely extending beyond the cervical third of the crown [16, 24]. The health status of the volunteers was self-reported. The exclusion criteria were as follows: 1) having oral diseases, such as periodontitis, salivary gland disorders, and oral mucosal diseases; 2) having autoimmune disease; 3) receiving any antibiotic treatment in the past three months; 4) a history of smoking history; 5) drinking tea, coffee, and other beverages with tannin in the past month; and 6) having surgical procedures during the several years prior to this study. In total, 26 healthy subjects without caries and pigment, 40 patients with pigment (black extrinsic stain)
(Additional file 1: Figure S1), 20 patients with caries (obvious decay), and 25 patients with both pigment (black extrinsic stain) and caries (obvious decay) in their primary dentition were recruited to donate samples on March 1, 2011 in Kunming, China. Multiple supragingival plaques were removed aseptically and pooled together from each patient or healthy subject. At the same time, saliva from each patient or healthy individual was extracted using 2-ml sterile needle tubing. All salivary and supragingival plaque samples were placed in cryovials without preservative, immediately snap frozen in liquid nitrogen, and stored at $-80{ }^{\circ} \mathrm{C}$ until bacterial community analysis.

\section{Saliva and supragingival plaque sample preparation and DNA extraction}

Each sample was suspended while frozen in a solution containing $200 \mu \mathrm{l}$ of buffer ATL and $200 \mu \mathrm{l}$ of a slurry of 0.1-mm diameter zirconia/silica beads (BioSpec Products, Bartlesville, OK). The mixed sample was then lysed by mechanical disruption with a bead beater (BioSpec Products) set on high for $2 \min \left(20^{\circ} \mathrm{C}\right)$, followed by extraction with a QIAamp DNA Stool Mini Kit (Qiagen, Hilden, Germany) according to its protocol for the isolation of DNA for pathogen detection. The DNA from each sample was eluted in a final volume of $200 \mu \mathrm{l}$ of elution buffer and stored at $-20{ }^{\circ} \mathrm{C}$.

\section{PCR amplification of the V1-V3 region of bacterial 16S rRNA genes}

Triplicate PCR reactions were performed for each sample. We referred previous studies $[25,26]$ for primer designs as follows: the forward primer (5'-CGTATCGCCTCCCT CGCGCCATCAGNNNNNNNAGAGTTTGATCMTGGC TCAG-3') contained the 454 Life Sciences primer A sequence, a unique 7-nt barcode used to tag each PCR product (designated by NNNNNNN) and the broad-range bacterial primer $8 \mathrm{~F}$. The reverse primer $\left(5^{\prime}\right.$-CTATGCGC CTTGCCAGCCCGCTCAGGTATTACCGCGGCTGCTG GCAC-3') contained the 454 Life Sciences primer B sequence and the broad-range bacterial primer 533R. Each $25-\mu \mathrm{l}$ reaction contained $0.2 \mu \mathrm{M}$ forward and reverse primers, $3 \mu \mathrm{l}$ of template DNA, $2.5 \mu \mathrm{l}$ of $10 \times$ PCR buffer plus $\mathrm{Mg}^{2+}$ (TaKaRa, Dalian, China), $2.0 \mu \mathrm{l}$ of dNTP (2.5 mM each) (TaKaRa), $0.75 \mu \mathrm{l}$ of DMSO (100\%), and $0.25 \mu \mathrm{l}$ of TaKaRa Taq ${ }^{T M}(5 \mathrm{U} / \mu \mathrm{l})$. All dilutions were performed using sterile $\mathrm{ddH}_{2} \mathrm{O}$. The PCR reactants were assembled in a PCR hood in which all surfaces and pipettes had been decontaminated by $30 \mathrm{~min}$ of autoclaving at $120{ }^{\circ} \mathrm{C}$ and $30 \mathrm{~min}$ of UV irradiation. Thermal cycling took place at $94{ }^{\circ} \mathrm{C}$ for $3 \mathrm{~min}$, followed by 5 cycles of $94{ }^{\circ} \mathrm{C}$ for $20 \mathrm{~s}, 45^{\circ} \mathrm{C}$ for $20 \mathrm{~s}$, and $65{ }^{\circ} \mathrm{C}$ for $60 \mathrm{~s}$ and then 20 cycles of $94{ }^{\circ} \mathrm{C}$ for $30 \mathrm{~s}, 58{ }^{\circ} \mathrm{C}$ for $20 \mathrm{~s}$, and $72{ }^{\circ} \mathrm{C}$ for $30 \mathrm{~s}$, with a final extension at $72{ }^{\circ} \mathrm{C}$ for $5 \mathrm{~min}$. Replicate amplicons were pooled and visualized 
on $1.5 \%$ agarose gels using EB stain in 0.5X TE. Amplicons were cleaned using a MinElute ${ }^{\circ}$ Gel Extraction Kit (Qiagen, Shanghai, China) according to the manufacturer's instructions. Next, we performed pyrosequencing using primer A and titanium chemistry on a 454 Life Sciences Genome Sequencer FLX instrument (Roche) at Sangon Biotech (Shanghai) Co., Ltd, China.

\section{Sequence data preparation and preliminary analysis}

Sequences were processed and analyzed using the Qiime pipeline [27]. After quality control with a minimum quality score of Q20 per base, the quality control sequence dataset was clustered into operational taxonomic units (OTUs) using denovo usearch61 [v6.1.544 (beta)] [28] with the criterion of a minimum identity of $97 \%$. The taxonomy of the OTUs was determined using BLAST $(\mathrm{E}$-value $=0.001$ ) against the Greengenes database from the August 2013 release [29]. Based on the identified OTU dataset and the corresponding taxonomic assignments, we constructed an OTU count table with the taxonomic information. We excluded singletons (one sequence per OTU) from the OTU table when performing the following analyses. Based on an rarefaction analysis, all samples were sub-sampled to the same read depth to limit the effects of differential sampling that are known to affect alpha and beta diversity metrics and to differentially increase the representation of PCR and sequencing artifacts in datasets. Finally, we created a table of counts for the number of times each OTU or genus-level phylotype was observed in each sample. Principal Coordinate Analysis (PCoA) in this study is used to compare groups of samples based on both unweighted Unifrac [30] and binary jaccard distance metrics.

\section{Community typing}

The resulting table was used as an input to partition all samples according to community types using the DMM $[2,3]$ and PAM [31] models supplied in mothur version 1.36.1 [32]. The analysis was performed at least ten times to confirm that we had obtained the minimum Laplace approximation used as the criteria for selecting the number of community types [3]. Samples were assigned to their community type based on the maximum posterior probability. Community types were visualized by non-metric dimensional scaling (NMDS) ordination of Bray-Curtis divergence values between oral samples using the DMM approach in mothur.

\section{Statistics}

General characteristics were represented as the mean or median. A hierarchically clustered heatmap analysis of oral samples was performed based on microbial abundances using average linkage with Pearson correlation, using the function aheatmap in Nonnegative Matrix
Factorization (NMF) $R$ package [33]. Multiple sample comparisons were performed using one-way analysis of variance (ANOVA) (parametric) or Kruskal-Wallis oneway ANOVA on ranks (non-parametric) using SigmaPlot 12.0 (Systat Software, Inc.). Based on the Bray-Curtis distance, the analysis of molecular variance (AMOVA) (determines whether the genetic diversity within two or more communities is greater than their pooled genetic diversity) and homogeneity of molecular variance (HOMOVA) (determines whether the amount of genetic diversity in each community is significantly different) provided by mothur version 1.36.1 [32] were used to compare the community structures. Co-occurring analysis using the Spearman rank correlation was conducted using Hmisc 3.9-3 (Harrell, Vanderbilt University School of Medicine, Nashville, TN, USA) in the R software package and the relative abundance of bacterial genera. Each co-occurring pair had an absolute value of Spearman rank correlation coefficient $(r)>0.50$ with statistical significance level under 0.0001 .

\section{Results}

We collected 111 saliva and 110 supragingival plaque samples from the primary dentition of 85 patients and 26 healthy subjects, yielding a total of 221 samples for molecular analysis of the bacterial $16 \mathrm{~S}$ rRNA genes. We obtained a dataset consisting of 831,381 high-quality $16 \mathrm{~S}$ rRNA gene sequences (the V1-V3 hypervariable regions) with an average of 3,762 \pm 165 (S.E.) sequences per sample. The PCoA analyses based on both unweighted Unifrac and binary jaccard measures consistently showed significant $(P=0.001$, anosim test $)$ segregation of salivary and plaque samples (Additional file 2: Figure S2A). To limit the effects of differential sampling and to render the microbial communities of samples comparable, we performed a rarefaction curve analysis (Additional file 3: Figure S3) by which we found that 2000 sequence subsampling was adequate to reflect the complex of oral microbiota because the increase of observed species tends to slow. Thus, to compare samples, all samples were sub-sampled to 2,000 reads following the removal of 23 salivary and 20 plaque samples with reads fewer than 2,000. The final 178 samples were used to create a table of counts for each OTU (or species)-level phylotype observed in each sample for PCoA-based community typing. The result still reflected significantly $(P=0.001$, anosim test) microbial community divergence dependent on the heterogeneity of salivary and plaque niches (Additional file 2: Figure S2B).

To discover disease-associated community-level changes of the oral microbial world, we performed DMM-based community typing based on genus-level phylotype observed in each of the 178 samples. We identified five community types supported by the smallest Laplace value after ten 
independent runs; these communities included two types $(A$ and $B)$ in plaque niches and three types $(C, D$, and $E$ ) in saliva, which was visualized by NMDS method (Additional file 4: Figure S4). Between $91 \%$ and $100 \%$ of the samples assigned into five community types had a posterior probability of at least 0.99 . Significant differences among and within five communities were further confirmed by both HOMOVA and AMOVA tests $(P<0.001)$. Furthermore, we found 20 genus-level taxa contributing to $72.55 \%$ of the total difference for assessing five community types with DMM approach (Fig. 1). All 178 samples were hierarchically clustered into similar five community groups based on relative abundances of those 20 genera (Fig. 1). We further compared the differences of the top ten genera contributing to $56.9 \%$ of the total differences among five community types (Fig. 2). The results found each of five types dominated by specific bacterial compositions: Rothia, Prevotella, and Streptococcus were the most dominant $(P<0.001$, Kruskal-Wallis one-way ANOVA on ranks) in type A, Neisseria and unclassified Streptococcaceae $(P<0.001)$ in type B, Leptotrichia $(P<0.001)$ in type $C$, Paenibacillus in type D, and Parascardovia and unclassified Neisseriaceae in type $\mathrm{E}(P<0.001)$.

To identify the oral microbial community types potentially associated with diseases, we compared sample distribution differences among community types within plaque or salivary niches (Fig. 3). First, there was no significant difference in the samples of the same sources between the two plaque community types, A and B. Second, for the three salivary community types (C-E), we found that for the salivary samples of healthy subjects, type $C$ was significantly more likely than type $\mathrm{D}(P<0.0001$, Fisher test) and type $\mathrm{E}(P<0.001)$, suggesting that type $C$ may be representative of healthy individuals (Fig. 3). In addition, for salivary samples of patients with mixed caries and pigment (CPS), type $\mathrm{D}$ was significantly $(P<0.05)$ more likely than type $E$, whereas for the salivary samples of patients who only had pigment (PS), type $\mathrm{E}$ was significantly $(P<0.05)$ more likely than in type D (Fig. 3). Such marginal statistic significances weakly supported the divergence of two salivary community types (D and E) likely associated with the presence of CPS and PS.

To further characterize disease-associated community types, we determined the dominant bacterial genera potentially associated with diseases from five community types. For type A (Fig. 4), three significantly and positively correlated bacterial genus pairs were separately identified in pigment-containing plaques (PP) (Leptotrichia and Fusobacterium), caries-active plaques (CP) (unclassified Gemellales and Granulicatella), and plaques with the occurrence of mixed caries and pigment (CPP) (Streptococcus and Mogibacterium). All these bacterial genera were significantly $(P<0.05-0.001$, Kruskal-Wallis one-way ANOVA on ranks) dominant in three disease types $\mathrm{PP}, \mathrm{CP}$, and $\mathrm{CPP}$, respectively. For type B (Fig. 5), three dominant $(P<0.05-$

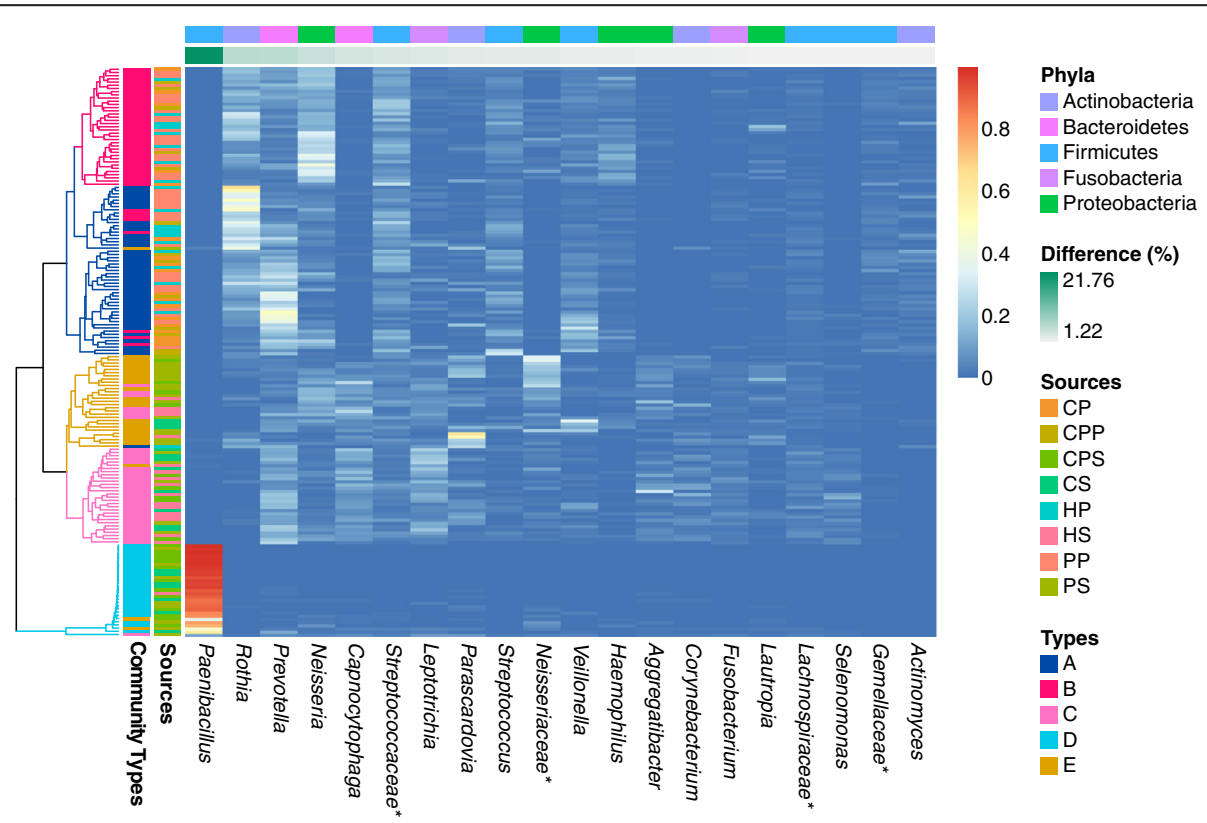

Fig. 1178 samples were hierarchically clustered into five groups based on oral bacterial genera using average linkage with Pearson correlation. The heat map shows relative abundance of top 20 bacterial genera (horizontal axis) in each sample (vertical axis) contributing to $72.55 \%$ of the total difference for assessing five community types with DMM approach. The stars denote unclassified taxa. HP: Healthy Plaque; CP: Caries-active Plaque; PP: Pigment-occurred Plaque; CPP: Caries + Pigment Plaque; HS: Healthy Saliva; CS: Caries-active Saliva; PS: Pigment-occurred Saliva; CPS: Caries + Pigment Saliva 

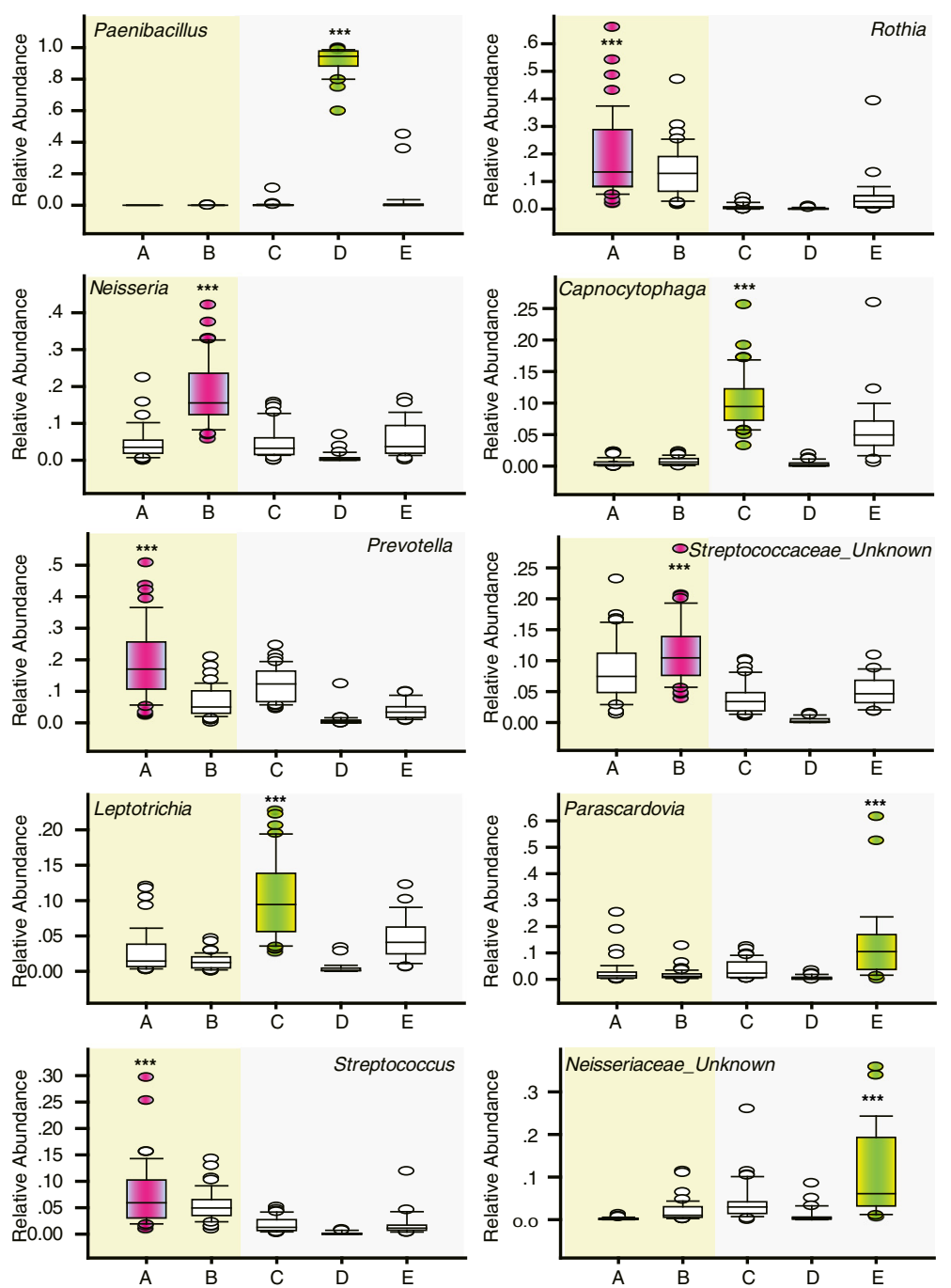

Fig. 2 Top ten bacterial genera contributing to community types. The labels located along the horizontal axis of each panel represent five oral microbial community types (A and B belong to plaque samples, and C-E belong to saliva samples). The relative abundance of the ten most abundant genera in the samples assigned to each of the types (the boxes represent the interquartile range (IQR), and the error bars represent the $95 \%$ confidence intervals; $n$ (community type A) $=45 ; n$ (community type B) $=45 ; n$ (community type C) $=37 ; n$ (community type D) $=26 ; n$ (community type E) $=25$ ). Statistical significance was evaluated using the Kruskal-Wallis one-way analysis of variance on ranks. $P$-values: ${ }^{* *}<0.001,{ }^{* *}<0.01$, and ${ }^{*}<0.05$

0.01, Kruskal-Wallis one-way ANOVA on ranks) bacterial genera (unclassified Clostridiaceae, Peptostreptococcus, and Clostridium) were significantly and positively correlated in CPP. Three dominant $(P<0.05-0.0001$, Kruskal-Wallis one-way ANOVA on ranks) bacterial genera (Selenomonas, Gemella, and Streptobacillus) were identified in CP but were not correlated. In addition, we did not find any difference for two salivary types $C$ and D in the bacterial composition across healthy subjects and patients. Consistent with those observations, HOMOVA test analysis found that those two community types had the minimum community diversity variations. For type $\mathrm{E}$, we found that $\mathrm{CS}$ was dominated $(P<0.01-0.001$, Kruskal-Wallis one-way ANOVA on ranks) by three significantly and positively correlated bacterial genera (Haemophilus, Veillonella, and Prevotella in the Paraprevotellaceae family) (Fig. 6). Totally, these findings suggested that two plaque type $\mathrm{A}$ and $\mathrm{B}$ as well as salivary type E may be associated with our concerned three disease types, although the lack of strong evidences from sample distributions across community types within salivary or plaque niches.

\section{Discussion}

For oral microbial community typing, we found that genus-based community typing (five types identified) using the DMM approach was superior to OTU-based analysis (two types inferred by PCoA in Additional file 2: Figures S2; three types by DMM in Additional file 4: Figure S4). Because our identified five community types not only reflected microbial community divergences 


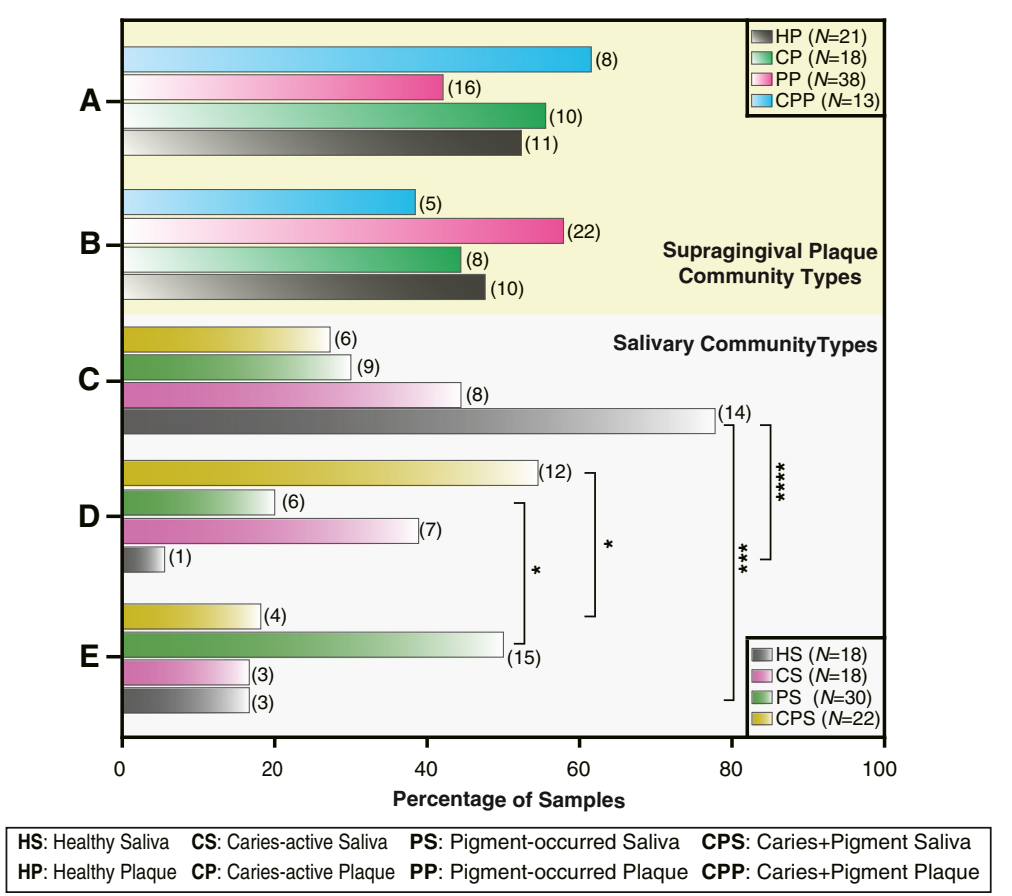

Fig. 3 Sample distribution comparisons across community types. The labels located along the vertical axis represent five oral microbial community types (a and $\mathbf{b}$ belong to plaque samples, and $\mathbf{c}$-e belong to saliva samples). Each bar represents the sample distribution percentage from different sources in each community type within the same niches. Sample number of each source assigned to each type placed to the right of the bar. Statistical significance was evaluated using Fisher's test. $P$-values: ${ }^{* * *}<0.0001,{ }^{* *}<0.0001,{ }^{* *}<0.01$, and ${ }^{*}<0.05$

driven by the heterogeneity of oral environmental niches (e.g., saliva and supragingival plaque), but also showed potential disease-associated community-level changes of oral microbiota within the same niche, such as two community types upon plaque. These results suggest that oral microbial diversity at the genus level can provide high-resolution information regarding the potential role of microbial infections in caries and pigment. We also found that both two plaque types and one salivary type dominated by specific bacterial genera may be associated with the occurrence of caries and pigment. Thus, further large-scale investigations combined with metadata describing changes in health or lifestyle would be interesting to reinforce those findings, because oral microbial diversities are greatly influenced by both infectious agents and other complex host factors, including lifestyle, genotype, physiology and the immune system [34]. Therefore, it is critical to use a statistically sound measurement of oral microbial diversity to distinguish infectious agents from other host factors.

Multiple distinct oral bacterial components between healthy subjects and patients were discovered from each plaque community type associated with our diseases of interest (Figs. 4 and 5). Conversely, only three correlated bacterial genera in saliva were found to be associated with caries (Fig. 6). These results suggest that plaque-associated microbial communities are much more effective than salivary microbial communities as microbial biomarkers for diagnosing and/or preventing caries and pigment. For instance, bacterial species belonging to Veillonella, Actinomyces, and Granulicatella are frequently found to contribute to dental caries [7, 35]. Consistently, our study demonstrated that a positive interplay $\left(R=0.81, P=2.0 \times 10^{-7}\right)$ between Granulicatella and unclassified Gemellales in plaque-community type A (Fig. 4) was closely related to the occurrence of caries. These findings tend to support the polymicrobial nature of oral infectious diseases [36]. In addition, from plaquecommunity type B, the independent overgrowth of Selenomonas, Gemella, and Streptobacillus was observed in caries-active patients (Fig. 5a). Of them, Selenomonas species were found to be common in advanced caries [37]. Gemella species have been isolated and observed experimentally to have high degrees of autoaggregation associated with the initiation and development of dental plaque and biofilms [38]. Recently, a Streptobacillus species has also been found to be a novel plaque bacterial phylotype in children younger than 30 months with caries [7]. Taken together, these findings suggest that plaque-associated infectious agents might play a critical role in the formation of caries in primary dentition. Notably, saliva-associated Veillonella was significantly more abundant in caries-active patients than in healthy subjects (Fig. 6). Haemophilus and Prevotella (Paraprevotellaceae) demonstrated consistent overgrowth 


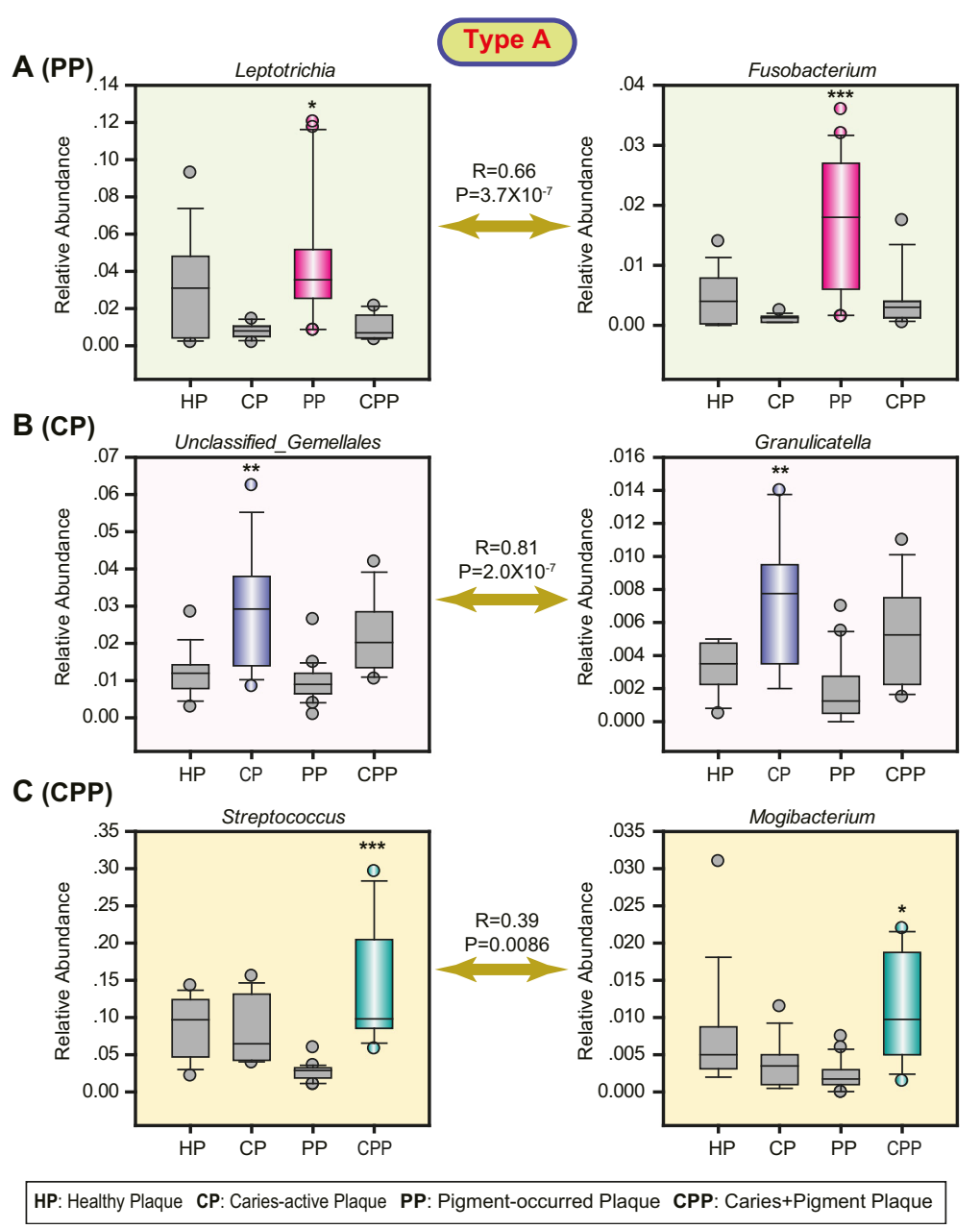

Fig. 4 Dominant bacterial genera are potentially associated with the presence of pigment (a), caries (b), and mixed caries/pigment (c) in the plaque-unique community type A. The relative abundance of the most abundant genera in the samples assigned to each of the sample types (the boxes represent the IQR, and the error bars represent the $95 \%$ confidence interval; $n(H P)=11 ; n(C P)=10 ; n(P P)=16 ; n(C P P)=8)$. Statistical significance was evaluated using the Kruskal-Wallis one-way analysis of variance on ranks with adjusted $P$-values: ${ }^{* *}<0.001,{ }^{* *}<0.01$, and ${ }^{*}<0.05$. The pairwise positive correlations marked by double-arrow lines were calculated using the Spearman rank-order correlation with the correlation coefficient $R$ and the $P$-values

with Veillonella. One previous study has confirmed that the central role of Veillonella sp. in multispecies community formation is a key element in facilitating the succession of species during the development of dental plaque in vivo [39]. These results suggest that the overgrowth of Veillonella species in saliva may indicate a high risk of dental caries in primary dentition.

Another important finding is that the co-overgrowth of Leptotrichia and Fusobacterium belonging to the bacterial phylum Fusobacteria in plaque might contribute to the formation of pigment in primary dentition (Fig. 4A). Correspondingly, a high $29.4 \%$ detection rate for Leptotrichia species was reported in dental plaque samples from patients with black extrinsic tooth staining [40]. Fusobacterium has also been identified in black stain samples [19]. F. periodontium, which was first isolated from advanced periodontitis lesions [41], is an opportunistic pathogenic bacterium of the mouth and other body sites [42]. Genome evidences from the human oral $L$. buccalis [43] demonstrate that this bacterium can express genes involved in producing beta-Lactam resistance (see the KEGG pathway map: http://www.genome.jp/dbgetbin/www_bget?lba:Lebu_0334), which could protect other penicillin-susceptible bacteria from penicillin by producing the enzyme beta-lactamase, such as Fusobacterium spp. [44]. These findings indicated that $L$. buccalis may promote the oral colonization of Fusobacterium spp. for further infection. Fusobacterium spp. can co-aggregate with black-pigmented anaerobes (Porphyromonas gingivalis and Prevotella nigrescens) [45]. Similarly, co-infecting or coculturing Pseudomonas aeruginosa can induce pigment production of Staphylococcus aureus [46]. These 


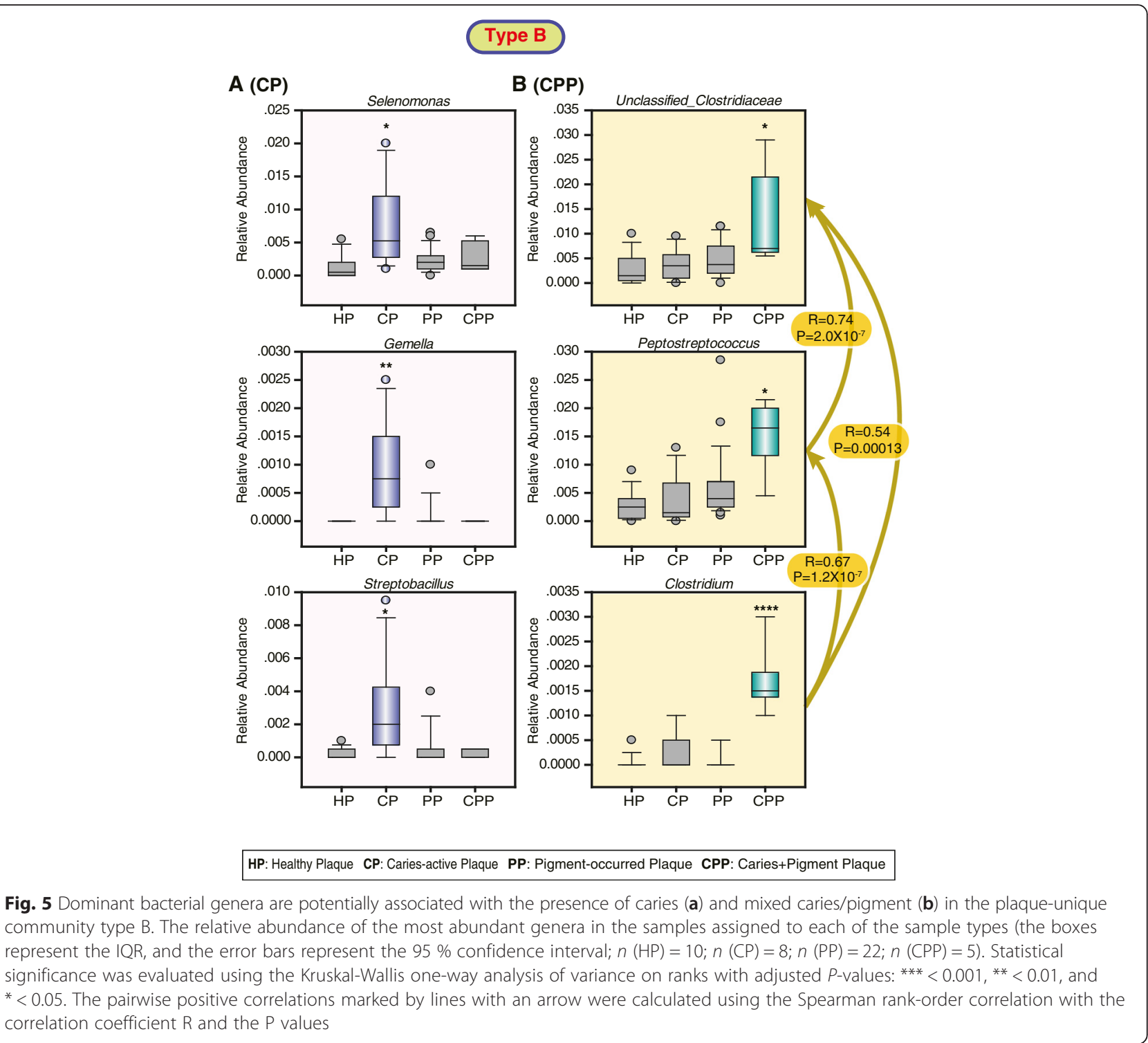

observations provide an important implication into understanding potential role of complex bacterial interactions in the formation of pigment. Thus, it is greatly expected to confirm these findings via future in vivo or in vitro studies. In addition, the co-occurrence of Leptotrichia and Fusobacterium was only observed in approximately half of the patients with pigment, suggesting that other factors, such as diet habit changes or/and human genotype differences, might also influence the formation of pigment.

The presence of black extrinsic tooth staining has been reported to be associated with low caries occurrence in affected patients compared with individuals who have healthy tooth surfaces [40]. Even so, this study suggested that two plaque-community types may be separately associated with the low-frequency presence of CPP. Our results suggest that Streptococcus and Mogibacterium co-infection in the plaque of patients may be associated with the presence of mixed pigment and caries (Fig. 4c). Streptococcus species are commonly associated with dental caries [7, 35]. The cariogenicity of Streptococci in gnotobiotic rats is well established [47]. Moreover, Mogibacterium species have been linked to human periodontal diseases [48] and the acute dental abscess [49]. These findings raise the possibility that Streptococcus and Mogibacterium co-infections may be associated with clinical indicators of both periodontal diseases and the mixed occurrence of caries and pigment. Meanwhile, our results also revealed a significantly positive correlation among Peptostreptococcus, Clostridium, and unclassified Clostridiaceae in plaque, which may contribute to the presence of CPP in human populations with the type B community (Fig. 5b). Peptostreptococcus spp. is detected in 


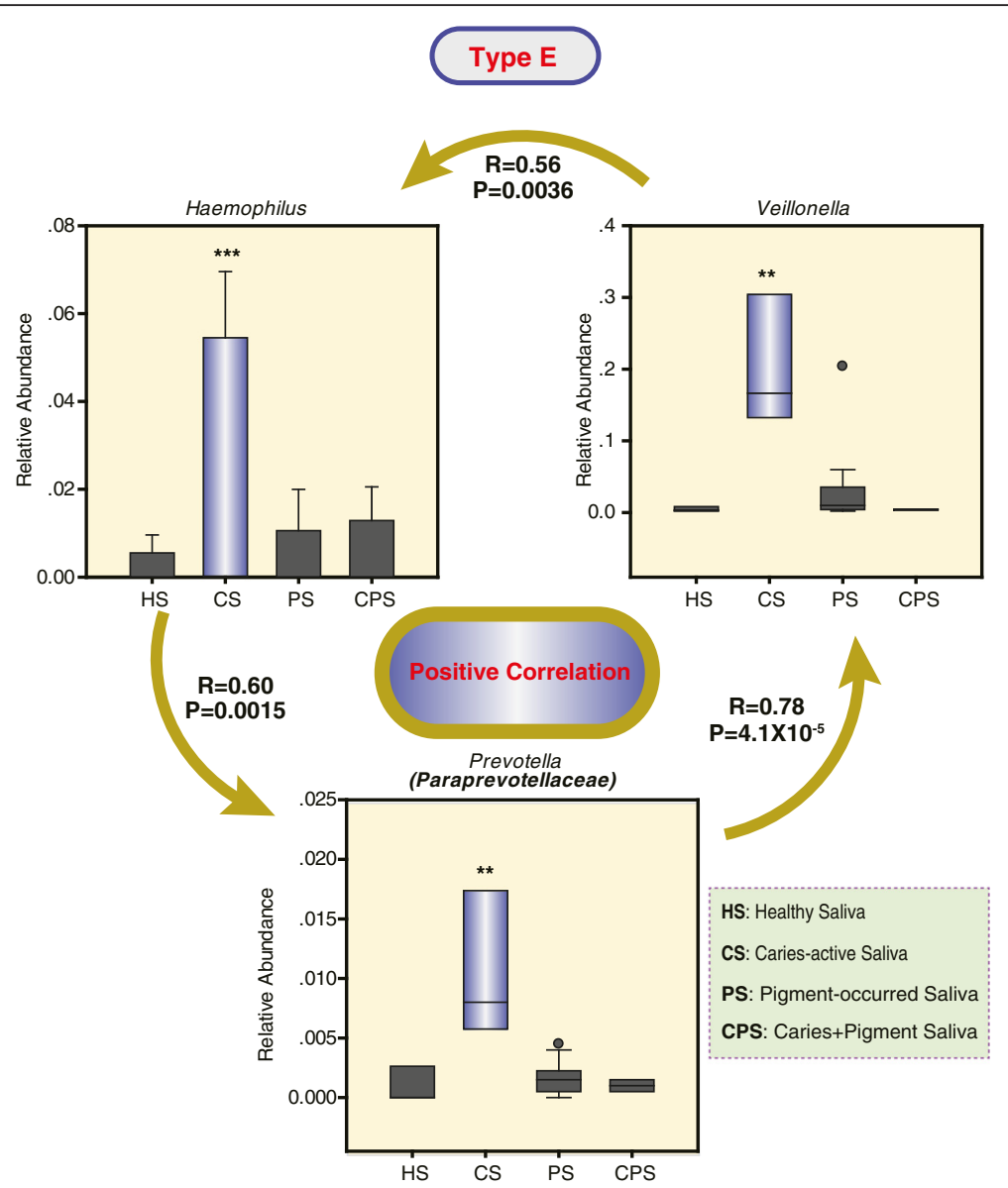

Fig. 6 Dominant bacterial genera are potentially associated with the presence of caries in the saliva-unique community type E. The relative abundance of the most abundant genera in the samples assigned to each of the sample types (the value for the genus Haemophilus is expressed as the mean \pm s.d.; the other two genera are shown with the IQR and the $95 \%$ confidence interval; $n(H S)=3 ; n(C S)=3 ; n(P S)=15 ; n$ (CPS) =4). Statistical significance was evaluated using Student's t-test (the genus Haemophilus) or the Kruskal-Wallis one-way analysis of variance on ranks (the other two genera) with adjusted $P$-values: ${ }^{* *}<0.001,{ }^{* *}<0.01$, and ${ }^{*}<0.05$. The positive correlations among three genera marked by lines with arrows were calculated using the Spearman rank-order correlation with the correlation coefficient $\mathrm{R}$ and the $P$ values

primary endodontic infections [50]. The cariogenicity of Peptostreptococci in gnotobiotic rats is also well established [47]. The Clostridia are reported from odontogenic infections [49]. These findings suggest that those bacteria associated with plaque CPP may contribute to the occurrence of caries or other oral diseases, although there was no evidence of an association with the combination of pigment and caries.

\section{Conclusions}

We propose the DMM approach is a good option to profile the human microbial communities associated with the human health or diseases based on $16 \mathrm{~S}$ rRNA gene sequence analysis. Our results indicate a distinct microbial community and composition in the oral cavities of patients with caries, pigment, or a mixture of caries and pigment, suggesting that the microflora associated with a variety of oral diseases is heterogeneous. The plaque- associated flora is much more effective than those in saliva as biomarkers for diagnosing and/or preventing caries and pigment. This information can be used as a basis for understanding the involvement of specific bacteria in the occurrence of caries and pigment.

\section{Additional files}

Additional file 1: Figure S1. Three typical cases from 40 patients with black-stained plaques. Y.HG.L., a specialist in pediatric dentistry, took responsibility for the black stain diagnosis and specimen collection. The clinical characteristics of all black-stained plaques as presented by three cases were softness and difficulty in removal. Supragingival black-stained plaque samples (including both the outside edge and the middle) from each patient with a typical black stain were pooled together for bacterial community analysis. (PPT $1583 \mathrm{~kb}$ )

Additional file 2: Figure S2. Principal coordinate analysis (PCOA) of microbial communities based on (A) 111 salivary and 110 supragingival plaque samples and (B) 88 salivary and 80 supragingival plaque samples after sub-sampled to 2,000 reads. Both unweighted Unifrac and binary 
jaccard measures showed consistent patterns. The anosim test is used to detect significant difference between saliva and plaque (See Methods). (PDF $746 \mathrm{~kb}$ )

Additional file 3: Figure S3. Rarefaction measures of bacterial $16 \mathrm{~S}$ rRNA sequences from 111 saliva and 110 supragingival plaque samples. The rarefaction curve based on observed species counting under different sequence depth is plotted by sub-sampling from 100 to 2000 with 100 sequence increase each step without any replacement following ten replicates. (PDF $188 \mathrm{~kb}$ )

Additional file 4: Figure S4. Community type assignment for nonmetric dimensional scaling (NMDS) ordination of Bray-Curtis divergence values of 178 samples after subsampling using the DMM approach based on genus and OTU abundances. The stress computed for this ordination was 0.24 , and the $R^{2}$ between the input distance matrix and the distance matrix calculated between the points for this ordination was 0.81 . (PDF $392 \mathrm{~kb}$ )

\section{Abbreviations}

16 rRNA, 16S ribosomal RNA; ANOVA, one-way analysis of variance; CP, caries-active plaque; CPP, caries + pigment plaque; $C P S$, caries + pigment saliva; CS, caries-active saliva; DMM, dirichlet multinomial mixture; HOMOVA homogeneity of molecular variance; HP, healthy plaque; $\mathrm{HS}$, healthy saliva; NMDS, non-metric dimensional scaling; NMF, nonnegative matrix factorization; OTUs, operational taxonomic units; PAM, partitioning around meloids; PCOA, principal coordinate analysis; PP, pigment-occurred plaque; PS, pigmentoccurred saliva

\section{Acknowledgements}

We thank Peng Shi (Kunming Institute of Zoology) for his help and suggestions for our data analysis.

\section{Funding}

This work was funded by support from a grant (No. 2012FB075) from the joint fund for the Yunnan Department of Science and Technology-Kunming Medical University.

\section{Availability of data and materials}

The dataset supporting the conclusions of this article is available in the NCBI SRA (http://trace.ncbi.nlm.nih.gov/Traces/sra/) under accession no. SRP058400.

\section{Authors' contributions}

YHIL contributed to data acquisition, analysis, and interpretation. CZ contributed to the study design. YF contributed to data analysis and interpretation. YHGL, ZQ and LB contributed to sample acquisition. ZZ and JL contributed to the conception and design of the study. YHIL, CZ, YF, YHGL, QZ and BL drafted the manuscript. ZZ and $J L$ critically revised the manuscript. All authors gave final approval and agree to be accountable for all aspects of the work, ensuring its integrity and accuracy.

\section{Competing interests}

The authors declare that they have no competing interests.

\section{Consent for publication}

Not applicable.

\section{Ethics approval and consent to participate}

The use of human subjects was approved by the Medical Ethics Board of Kunming Medical University, China. All participants signed written informed consent forms. The methods were conducted in accordance with the approved guidelines.

\section{Author details}

'Department of Preventive \& Pediatric Dentistry, The Affiliated Stomatology Hospital of Kunming Medical University, Kunming, Yunnan 650100, China. ${ }^{2}$ State Key Laboratory for Conservation and Utilization of Bio-Resources in Yunnan, Yunnan University, Kunming, Yunnan 650091, China. ${ }^{3}$ Department of Dermatology, Beijing Hospital, Beijing 100730, China. ${ }^{4}$ State Key Laboratory of Genetic Resources and Evolution, Kunming Institute of Zoology, Chinese Academy of Sciences, Kunming 650223, China.
Received: 12 April 2016 Accepted: 6 July 2016

Published online: 05 August 2016

\section{References}

1. Arumugam M, Raes J, Pelletier E, Le Paslier D, Yamada T, Mende DR, Fernandes GR, Tap J, Bruls T, Batto J-M, et al. Enterotypes of the human gut microbiome. Nature. 2011:473(7346):174-80.

2. Holmes I, Harris K, Quince C. Dirichlet Multinomial Mixtures: Generative Models for Microbial Metagenomics. PLoS One. 2012;7(2):e30126.

3. Ding T, Schloss PD. Dynamics and associations of microbial community types across the human body. Nature. 2014;509(7500):357-60.

4. Jiang $W$, Zhang J, Chen $H$. Pyrosequencing analysis of oral microbiota in children with severe early childhood dental caries. Curr Microbiol. 2013;67(5):537-42.

5. Luo AH, Yang DQ, Xin BC, Paster BJ, Qin J. Microbial profiles in saliva from children with and without caries in mixed dentition. Oral Dis. 2012;18(6):595-601.

6. Gomar-Vercher S, Cabrera-Rubio R, Mira A, Montiel-Company JM, AlmerichSilla JM. Relationship of children's salivary microbiota with their caries status: a pyrosequencing study. Clinical Oral Invest. 2014;18(9):2087-94.

7. Xu H, Hao W, Zhou Q, Wang W, Xia Z, Liu C, Chen X, Qin M, Chen F. Plaque bacterial microbiome diversity in children younger than 30 months with or without caries prior to eruption of second primary molars. PLoS One. 2014;9(2):e89269.

8. Xu X, He J, Xue J, Wang Y, Li K, Zhang K, Guo Q, Liu X, Zhou Y, Cheng L, et al. Oral cavity contains distinct niches with dynamic microbial communities. Environ Microbiol. 2015;17(3):699-710.

9. Teng F, Yang F, Huang S, Bo C, Xu Zhenjiang Z, Amir A, Knight R, Ling J, Xu J. Prediction of early childhood caries via spatial-temporal variations of oral microbiota. Cell Host Microbe. 2015;18(3):296-306.

10. Belda-Ferre P, Alcaraz LD, Cabrera-Rubio R, Romero H, Simon-Soro A, Pignatelli M, Mira A. The oral metagenome in health and disease. ISME J. 2012;6(1):46-56

11. Koleoso DC, Shaba OP, Isiekwe MC. Extrinsic tooth discolouration in 11-16 year-old Nigerian children. Odontostomatol Trop. 2004;27(106):29-34.

12. Gasparetto A, Conrado CA, Maciel SM, Miyamoto EY, Chicarelli M, Zanata RL. Prevalence of black tooth stains and dental caries in Brazilian schoolchildren. Braz Dent J. 2003:14(3):157-61.

13. Koch MJ, Bove M, Schroff J, Perlea P, Garcia-Godoy F, Staehle HJ. Black stain and dental caries in schoolchildren in Potenza, Italy. ASDC J Dent Child. 2001;68(5-6):353-5. 302.

14. Heinrich-Weltzien R, Monse B, Van Palenstein HW. Black stain and dental caries in Filipino schoolchildren. Community Dent Oral. 2009;37(2):182-7.

15. Garcia Martin JM, Gonzalez Garcia M, Seoane Leston J, Llorente Pendas S, Diaz Martin JJ, Garcia-Pola MJ. Prevalence of black stain and associated risk factors in preschool Spanish children. Pediatr Int. 2013;55(3):355-9.

16. Hattab FN, Qudeimat MA, Al-Rimawi HS. Dental discoloration: an overview. J Esthet Restor Dent. 1999:11(6):291-310.

17. Reid JS, Beeley JA. Biochemical studies on the composition of gingival debris from children with black extrinsic tooth stain. Caries Res. 1976;10(5):363-9.

18. Theilade J, Slots J, Fejerskov O. The ultrastructure of black stain on human primary teeth. Scand J Dent Res. 1973;81(7):528-32.

19. Slots J. The microflora of black stain on human primary teeth. Eur J Oral Sci. 1974;82(7):484-90.

20. Saba C, Solidani M, Berlutti F, Vestri A, Ottolenghi L, Polimeni A. Black stains in the mixed dentition: a PCR microbiological study of the etiopathogenic bacteria. J Clin Pediatr Dent. 2006:30(3):219-24.

21. Reid JS, Beeley JA, MacDonald DG. Investigations into black extrinsic tooth stain. J Dent Res. 1977:56(8):895-9.

22. Costa MT, Dorta ML, Ribeiro-Dias F, Pimenta FC. Biofilms of black tooth stains: PCR analysis reveals presence of Streptococcus mutans. Braz Dent J. 2012;23(5):555-8.

23. Pitts NB, Ekstrand KR, The IF. International Caries Detection and Assessment System (ICDAS) and its International Caries Classification and Management System (ICCMS) - methods for staging of the caries process and enabling dentists to manage caries. Community Dent Oral. 2013:41(1):e41-52.

24. Brook AH, Smith RN, Lath DJ. The clinical measurement of tooth colour and stain. Int Dent J. 2007:57(5):324-30.

25. Lazarevic V, Whiteson K, Hernandez D, Francois P, Schrenzel J. Study of inter- and intra-individual variations in the salivary microbiota. BMC Genomics. 2010;11(1):523.

26. Baker GC, Smith Jل, Cowan DA. Review and re-analysis of domain-specific 165 primers. J Microbiol Meth. 2003;55(3):541-55. 
27. Caporaso JG, Kuczynski J, Stombaugh J, Bittinger K, Bushman FD, Costello EK, Fierer N, Pena AG, Goodrich JK, Gordon Jl, et al. QIIME allows analysis of high-throughput community sequencing data. Nat Meth. 2010;7(5):335-6.

28. Edgar RC. Search and clustering orders of magnitude faster than BLAST. Bioinformatics. 2010;26(19):2460-1.

29. DeSantis TZ, Hugenholtz P, Larsen N, Rojas M, Brodie EL, Keller K, Huber T, Dalevi D, Hu P, Andersen GL. Greengenes, a chimera-checked 16S rRNA gene database and workbench compatible with ARB. Appl Environ Microb. 2006;72(7):5069-72.

30. Lozupone C, Knight R. UniFrac: a new phylogenetic method for comparing microbial communities. Appl Environ Microbiol. 2005;71(12):8228-35.

31. Koren O, Knights D, Gonzalez A, Waldron L, Segata N, Knight R, Huttenhower C, Ley RE. A guide to enterotypes across the human body: meta-analysis of microbial community structures in human microbiome datasets. PLoS Comput Biol. 2013;9(1):e1002863.

32. Schloss PD, Westcott SL, Ryabin T, Hall JR, Hartmann M, Hollister EB, Lesniewski RA, Oakley BB, Parks DH, Robinson CJ, et al. Introducing mothur: Open-source, platform-independent, community-supported software for describing and comparing microbial communities. Appl Environ Microb. 2009;75(23):7537-41.

33. Gaujoux $\mathrm{R}$, Seoighe $\mathrm{C}$. A flexible R package for nonnegative matrix factorization. BMC Bioinformatics. 2010;11(1):1-9.

34. Turnbaugh PJ, Ley RE, Hamady M, Fraser-Liggett CM, Knight R, Gordon I. The human microbiome project. Nature. 2007:449(7164):804-10.

35. Ling Z, Kong J, Jia P, Wei C, Wang Y, Pan Z, Huang W, Li L, Chen H, Xiang C. Analysis of oral microbiota in children with dental caries by PCR-DGGE and barcoded pyrosequencing. Microb Ecol. 2010;60(3):677-90.

36. Darveau RP. Periodontitis: a polymicrobial disruption of host homeostasis. Nat Rev Micro. 2010;8(7):481-90.

37. Preza D, Olsen I, Aas JA, Willumsen T, Grinde B, Paster BJ. Bacterial profiles of root caries in elderly patients. J Clin Microbiol. 2008:46(6):2015-21.

38. Shen S, Samaranayake LP, Yip HK. Coaggregation profiles of the microflora from root surface caries lesions. Arch Oral Biol. 2005;50(1):23-32.

39. Periasamy S, Kolenbrander PE. Central role of the early colonizer veillonella sp. In establishing multispecies biofilm communities with initial, middle, and late colonizers of enamel. J Bacteriol. 2010;192(12):2965-72.

40. Takashima Y, Matsumi Y, Yamasaki Y, Hirano K, Yanagida K, Fujita K, Matsumoto-Nakano M. Black pigmentation in primary dentition: Case report and literature review. Pediatric Dental J. 2014;24(3):184-8.

41. Slots J, Potts TV, Mashimo PA. Fusobacterium periodonticum, a new species from the human oral cavity. J Dent Res. 1983;62(9):960-3.

42. Park S-N, Park J-Y, Kook J-K. Development of species-specific polymerase chain reaction primers for detection of Fusobacterium periodonticum. Microbiol Immunol. 2010;54(12):750-3.

43. Ivanova N, Gronow S, Lapidus A, Copeland A, Glavina Del Rio T, Nolan M, Lucas S, Chen $\mathrm{F}$, Tice $\mathrm{H}$, Cheng JF, et al. Complete genome sequence of Leptotrichia buccalis type strain (C-1013-b). Stand Genomic Sci. 2009;1(2):126-32.

44. Brook I. The role of beta-lactamase-producing-bacteria in mixed infections. BMC Infect Dis. 2009;9(1):1-4.

45. Bradshaw DJ, Marsh PD, Watson GK, Allison C. Role of Fusobacterium nucleatum and coaggregation in anaerobe survival in planktonic and biofilm oral microbial communities during aeration. Infect Immun. 1998;66(10):4729-32.

46. Antonic V, Stojadinovic A, Zhang B, Izadjoo MJ, Alavi M. Pseudomonas aeruginosa induces pigment production and enhances virulence in a white phenotypic variant of Staphylococcus aureus. Infect Drug Resist. 2013;6:175-86.

47. Subramaniam $P$, Konde $S$, Prashanth $P$. An in vitro evaluation of $\mathrm{pH}$ variations in calcium hydroxide liners. J Indian Soc Pedod Prev Dent. 2006;24(3):144-5.

48. Colombo APV, Boches SK, Cotton SL, Goodson JM, Kent R, Haffajee AD, Socransky SS, Hasturk H, Van Dyke TE, Dewhirst F, et al. Comparisons of subgingival microbial profiles of refractory periodontitis, severe periodontitis, and periodontal health using the human oral microbe identification microarray. J Periodontol. 2009;80(9):1421-32.

49. Robertson D, Smith AJ. The microbiology of the acute dental abscess. J Med Microbiol. 2009;58(2):155-62.

50. Siqueira Jr JF, Rôças IN, Andrade AFB, de Uzeda M. Peptostreptococcus micros in primary endodontic infections as detected by $16 \mathrm{~S}$ rDNA-based Polymerase Chain Reaction. J Endod. 2003;29(2):111-3. 